\title{
Los Factores Claves de la Competitividad en el Sector Hotelero: una Propuesta desde la Teoría de los Recursos y Capacidades (VBR)
}

\section{The Key Factors of Competitiveness in the Hotel Sector: a Proposal from the Theory of Resources and Capabilities (VBR)}

\author{
María A. Santis-Puche \\ Corporación Universitaria Antonio José de Sucre - \\ Colombia \\ ORCID iD: https://orcid.org/0000-0002-4366-7118 \\ Docente administración2@corposucre.edu.co
}

Fecha de recepción: 13/03/2021

Fecha de evaluación: 24/04/2021

Fecha de aceptación: 22/05/2021

Cómo citar: Santis-Puche, M. (2021). Los factores claves de la competitividad en el sector hotelero: una propuesta desde la teoría de los recursos y capacidades (VBR). Revista Científica Anfibios, 4(1). https://doi.org/10.37979/afb.2021v4n1.84

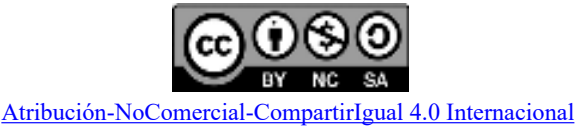

\section{Resumen}

En la actualidad, la competitividad ocupa una de las discusiones centrales en el ámbito académico y empresarial, respecto a cuáles son los factores que la afectan y permiten a las organizaciones diferenciarse de su competencia y generar una ventaja competitiva en el mercado. En este contexto, la teoría de los recursos y las capacidades (VBR) ha surgido como una corriente alternativa que basa la fuente de competitividad considerando las habilidades y las dimensiones internas de la organización. Entre ellas, se ha expuesto que son factores como el marketing, gestión, innovación calidad y talento humano aquellos que puedan tener un impacto directo en la competitividad de las organizaciones dedicadas a servicios hoteleros. Es así que se propone, desde un enfoque formativo, un análisis factorial confirmatorio que permita dilucidar los factores claves que afectan la competitividad en el sector hotelero. Para tales fines, se plantean unas encuestas en el sector hotelero de Sucre arrojando como resultado que son los factores del talento humano y la innovación aquellos que posibilitan la construcción de competitividad en este sector y en menor medida el marketing, la dirección y la calidad. A partir de estos resultados se plantean unas discusiones respecto a la importancia de los factores claves en la competitividad del sector.

\section{Palabras Clave}

Competitividad; visión basada en recursos; sector hotelero; innovación; talento humano

\begin{abstract}
Competitiveness is currently one of the central discussions in academia and business regarding the factors that affect it and allow organisations to differentiate themselves from their competitors and generate a competitive advantage in the market. In this context, the theory of resources and capabilities (VBR) has emerged as an alternative current that bases the source of competitiveness on the skills and internal dimensions of the organisation. Among these, it has been argued that factors such as marketing, management, innovation, quality and human talent can have a direct impact on the competitiveness of organisations dedicated to hotel services. Thus, from a formative approach, a confirmatory factor analysis is proposed to elucidate the key factors that affect competitiveness in the hotel sector. To this end, surveys are carried out in the hotel sector in Sucre, with the result that the factors of human talent and
\end{abstract}


innovation are those that make it possible to build competitiveness in this sector, and to a lesser extent marketing, management and quality. Based on these results, discussions are held on the importance of the key factors in the competitiveness of the sector.

\section{Keywords}

Competitiveness; resource based view; hotel sector; innovation; human talent

\section{Introducción}

En el entorno empresarial actual, la competitividad se ha convertido en un aspecto relevante de la dirección estratégica de las organizaciones debido a que está significativamente relacionada con el crecimiento empresarial, el desempeño organizacional y su proceso para lograrlos (Carmona-De Ríos, 2018). En ese sentido, las organizaciones están cada vez más interesadas en convertirse en líderes de su mercado, con buena reputación y obteniendo altos retornos financieros; sin embargo, para que una empresa obtenga estos niveles de competitividad, se ve afectada por una variedad de factores exógenos y endógenos, siendo compleja la identificación de los recursos internos como fuente de ventaja competitiva y un trabajo arduo el poder administrarlos adecuadamente (Del Río Cortina et al., 2018). Esto es especialmente característico del sector hotelero en donde son factores internos basados en el compromiso de los empleados y orientados al cliente, entre otros, los que ejercen una influencia positiva en la competitividad de estas organizaciones (Ruizalba Robledo et al., 2015).

En este contexto, la teoría Basada en Recursos (VBR) establece supuestos fundamentado en la heterogeneidad de las organizaciones, en un intento por responder al cuestionamiento de por qué empresas que compiten en el mismo sector $\mathrm{o}$ industria obtienen diferencias significativas en sus resultados (Martínez Santa et al., 2010). Esta teoría se basa en dos principios. Primero, cada empresa es heterogénea internamente, en la medida que existen diferencias respecto a los recursos estratégicos que controlan. Segundo, estas diferencias son relativamente estables, manteniendo la heterogeneidad a largo plazo, debido a la dificultad para transferir recursos de una empresa a otra, de manera que se haga sostenible la ventaja competitiva.

Estas consideraciones permiten un análisis de las fuentes de ventaja competitiva, entendiendo que la teoría asume que el resultado que desea toda empresa es lograr una ventaja competitiva sostenible (Martínez Santa et al., 2010); para lo cual, deben a partir de sus recursos desarrollar una serie de capacidades esenciales distintivas que le permitan lograr tal objetivo, como núcleo central del éxito y resultado organizacional (Mazaira Castro et al., 2005).

Ante este panorama, se requiere profundizar respecto a cuáles son los factores claves que intervienen en la competitividad de las organizaciones pertenecientes al sector hotelero, es por esto que se propone desde un enfoque formativo, establecer un análisis factorial confirmatorio que determine en qué medida afectan los factores internos de la organización, desde la teoría Basada en Recursos (VBR), contribuyendo al análisis de la competencia en esos escenarios.

Para estos fines, se parte de unos referentes teóricos en donde se expongan la competitividad, así como los principales factores claves que de acuerdo con la VBR podrían incidir en la competitividad. Posteriormente se plantean los planteamientos metodológicos haciendo énfasis en el análisis factorial confirmatorio como herramienta estadística que permita comprobar en qué medida afectan los factores del VBR a la competitividad, lo cual se planteara en los resultados y finalmente se expondrán algunas conclusiones y discusiones.

\section{Referentes Teóricos}

En la actualidad, la conceptualización de competitividad sigue generando interés tanto por la academia y como en el ámbito empresarial, no obstante, es una noción que viene discutiéndose desde hace mucho tiempo. Algunas corrientes teóricas consideran que la competitividad procede de los planteamientos clásicos de la economía postulados por pensadores como Adam Smith y David Ricardo considerando la temática de la competencia, formando un concepto fundamental e imprescindible para la teoría económica. 
En este marco, Adam Smith, precursor de la economía moderna, expreso algunos argumentos que en la actualidad podrían ser considerados dentro de lo que se entiende por competitividad y en contraposición, cuestionó las ideas de los mercantilistas sobre el control de la economía. Luego, David Ricardo, otro precursor de la economía, destaca la teoría de la ventaja comparativa, defendiendo las ventajas del comercio internacional como una ampliación de la división del trabajo propuesta por Adam Smith, oponiéndose a las teorías proteccionistas que defendían la producción del propia del país y evitaban el comercio exterior. De acuerdo a Ricardo, un territorio tiene ventajas comparativas en la producción si el costo de oportunidad de producir ese bien en términos de otros bienes es inferior en ese territorio respecto a otros" (Abdel \& Romo, 2005). Es así que la ventaja comparativa es impulsada por las diferencias en los costos de los insumos como la mano de obra o el capital.

Mas adelante, Joseph Schumpeter, expresa que la competencia entre empresas se refiere a la integración de nuevos productos y procesos, promoviendo el concepto de destrucción creativa como una forma de describir el proceso de transformación que acompaña a la innovación, y reemplazar nuevos métodos y productos liderados por grandes empresas por nuevos métodos y productos contribuyendo así al desarrollo del concepto de competitividad hasta entonces.

Sin embargo, es hasta la década de los sesenta que surgen los trabajos de Michael Porter, considerado actualmente el padre de la estrategia competitiva despertando gran interés sobre el tema, aportando nociones como el traslado del concepto de ventaja competitiva a las naciones, aplicado hasta entonces solo para empresas, sosteniendo además que los países pueden construir sus propias ventajas competitivas.

En su investigación, Porter expresa que las ventajas comparativas estáticas no son elementos determinantes para el incremento de la producción económica, puesto que un territorio tiene la posibilidad de generar sus propias ventajas por medio del desarrollo de nuevas aplicaciones tecnológicas, practicas gerenciales, infraestructura concebida para estos fines, entre otras; debatiendo las propuestas de Ricardo. Además, señala que, las organizaciones de un país deben pasar de competir con ventajas comparativas basadas en mano de obra o recursos naturales de bajo costo a competir con ventajas competitivas generadas por productos y procesos únicos. (Porter M. E., 1990).

En esta línea, Michael Porter, propone un marco teórico con la finalidad de analizar holísticamente los distintos sectores y actores de la industria, por medio del planteamiento de cinco fuerzas que intervienen en un sector industrial; basadas en los elementos claves del mercado: clientes, competidores directos, proveedores, productos sustitutos y competidores potenciales (Baena, Sánchez, \& Montoya, 2003).

Estas cinco fuerzas se refieren a: 1. La amenaza de entrada de nuevas empresas, 2. La amenaza de productos o servicios, 3. El poder negociador de los compradores, 4 . El poder negociador de los proveedores y 5 . La rivalidad existente entre los distintos competidores del sector.

En este sentido, este modelo de cinco fuerzas permite a una empresa descubrir un sector industrial prometedor antes de que se refleje en los precios de las empresas que puede adquirir; además, facilita la búsqueda de valiosos tipos de conexiones entre empresas diversificadas. Por lo tanto, el objetivo de formular una estrategia competitiva a través de este modelo es establecer un retorno de la inversión tomando acciones defensivas u ofensivas, de manera que la empresa pueda establecerse en la industria para enfrentar con éxito estas cinco fuerzas competitivas (Porter M. , 2015), es así que se plantea que una de las estrategias para competir en ese entorno radica en potencializar las habilidades internas de la organización.

Tomando en consideración lo anterior, la teoría VBR plantea el rol de los recursos y capacidades como elemento esencial de la estrategia empresarial y como los antecedentes principales de la rentabilidad, trasladando la atención desde la orientación sectorial prevaleciente hasta los 80 's y principios de los 90's, a la relación entre estrategia y el ámbito interno de la empresa (Suárez \& Ibarra, 2002; Barney, 1991, pág. 101).

En esta línea, la VBR esboza que para lograr y conservar una ventaja competitiva Las organizaciones deben contar con ciertos recursos clave con características específicas, porque no todos 
los recursos tienen el mismo potencial para convertirse en una fuente de ventaja competitiva. Las condiciones o características específicas establecidas son: tienen valor; son difíciles de imitar; y permiten la distribución de beneficios. (Barney, 1991; Lippman \& Rumelt, 1982; Peteraf, 1993; Collis \& Montgomery, 1995).

Dicho de otro modo, los recursos internos capaces de conferir ventajas competitivas a las organizaciones deben ser valioso, raro o escaso, no completamente imitable e irremplazables (Medina R. , 2013). De esta forma, las características se centran en la generación de ingresos, la sostenibilidad de dichos ingresos en el tiempo y la asignación de estos ingresos por parte de la empresa. (Martinez Santa, Charteriana Abando, \& Araujo de la Mata, 2010).

En este sentido la VBR enfatiza la importancia de la elección estratégica, como responsabilidad de la gerencia, su tarea es identificar, desarrollar y desplegar recursos clave para maximizar los beneficios de la organización; resalta la relevancia de los elementos internos de la empresa y conocer las razones de las diferencias en los resultados entre organizaciones, haciendo de la empresa una unidad de análisis (Martinez et al., 2010).

En este marco, Si bien no existe consenso en la literatura a la hora de determinar diversos factores o fuentes internas de competitividad, los estudios han mostrado factores internos que explican las diferencias de competitividad en un mismo sector. (Aragón et al., 2010); entre los cuales resaltan: la innovación (Lloyd-Reason, et al., 2002; Li, 2000); la calidad del producto o servicio (Aragón \& Rubio, 2005), la dirección y desarrollo de los recursos humanos (Warren \& Hutchinson, 2000; Li, 2000); la capacidad del directivo para gestionar la empresa (Camisón, 1997; Rogoff et al., 2004) y las competencias en marketing, (Li, 2000; Aragón \& Rubio, 2005), como se puede apreciar en la siguiente tabla.

Tabla 1. Factores internos como fuentes de ventaja competitiva

\begin{tabular}{ll}
\hline \multicolumn{1}{c}{ Factor } & \multicolumn{1}{c}{ Autores } \\
\hline \hline Marketing & Li, (2000); Aragón \& Rubio, 2005; Martínez Santa et al., 2010 \\
\hline Dirección & Camisón, (1997); Warren \& Hutchinson, (2000); Li, (2000); Rogoff, et al., (2004); Ara- \\
& gón \& Rubio, (2005) \\
\hline Innovación & (Li, 2000); (Aragón \& Rubio, 2005); (Lloyd-Reason, Muller, \& Wall, 2002); (Martínez \\
& Santa, Charteriana Abando, \& Araujo de la Mata, 2010); \\
\hline Calidad & (Aragón \& Rubio, 2005); (Martínez Santa, Charteriana Abando, \& Araujo de la Mata, \\
& 2010); \\
\hline Recurso / Talento Humano & (Li, 2000); (Aragón \& Rubio, 2005); (Martínez Santa, Charteriana Abando, \& Araujo \\
& de la Mata, 2010) \\
\hline \hline
\end{tabular}

Fuente: elaboración propia

Cabe señalar que otros factores son reconocidos en la literatura; no obstante, considerando los objetivos propuestos, desde la VBR, los previamente enfatizados serán considerados como impulsores de la competitividad

Las capacidades de marketing se refieren a que una organización reconocida se distingue en el mercado competitivo por una orientación al mercado y hacia él y sus clientes (Verhees \& Meulenberg, 2004; Pelham, 1997); en el que comprender sus deseos y brindar una mejor manera que la competencia es la clave de la ventaja competitiva (Conant et al., 1990; Hill, Jones, \& Schilling, 2014).
En este marco, existe una literatura que demuestra que los recursos de marketing son uno de los elementos con las características de crear ventajas competitivas, las cuales pueden entenderse como recursos utilizados en las actividades de marketing o generados por estas. (Martinez Santa et al., 2010). Enfatizando en recursos de marketing como la reputación de la marca, las relaciones con los clientes o la orientación al mercado, permite proponer la siguiente hipótesis.

$\mathrm{H}_{1}$ : EI Factor Interno De Marketing Tiene Una Influencia Positiva En La Ventaja Competitiva De Las Organizaciones Del Sector Hotelero. 
Por su parte, para la capacidad de dirección o recursos directivos muchos autores han demostrado la relevancia de este recurso para la generación y mantenimiento del éxito comercial. Este factor está relacionado con el diseño de la toma de decisiones, la planificación de objetivos, la elección estratégica y los medios de implementación. (Bañón Rubio \& Sanchez Aragón, 2005).

Martinez Santa et al., (2010) citando a Castanias \& Helfat (1991); Mahoney \& Pandian, (1992); Kor y Mahoney, (2000) expresan la importancia del rol que juegan los gerentes en la elección del camino que seguirá la organización, la combinación de recursos que se desplegarán y promoverán, y el mercado en el que participarán. Además, los autores afirman que los recursos de gestión son un catalizador para otros recursos porque representa un punto de apoyo para el desarrollo de otras capacidades.

En ese marco, al revisar la literatura sobre los aspectos a considerar a la hora de determinar las capacidades del gerente o un equipo directivo, se refieren a sus conocimientos, habilidades, destrezas, valores, pericia, actitudes, experiencia y habilidades; además de indicar la formación y desarrollo formal del gerente, aspectos innatos o cognitivos y sus experiencia acumulada es la base del conjunto de conocimientos y habilidades necesarios son esos aspectos que deben ser tenidos en cuento(Barney, 1991). Desde otra perspectiva, su nivel de educación, (Wiersema \& Bantel, 1992), experiencias laborales y las características particulares (Fondas \& Wiersema, 1997) engloban las capacidades directivas, dando origen a la siguiente hipótesis:

$\mathrm{H}_{2}$ : El Factor Interno De La Dirección Tiene Una Influencia Positiva En La Ventaja Competitiva De Las Organizaciones Del Sector Hotelero.

La Innovación o capacidad de innovación está definida por el Manual de Oslo (OCDE, 2005) como "la introducción de un nuevo, o significativamente mejorado, producto (bien o servicio), proceso, método de comercialización o método organizativo en las prácticas internas de la empresa, la organización del lugar de trabajo o las relaciones exteriores".

Galende \& Suárez (1999) expresan que el conocimiento técnico y las capacidades de inno- vación son uno de los recursos más importantes de una organización, así como una fuente importante de ventaja competitiva. Aragón \& Rubio (2005) establecen que El éxito de las empresas depende de su capacidad para adaptarse mejor a los entornos dinámicos, por lo que necesitan mecanismos que impulsen la innovación para responder a los cambios en la tecnología, los productos y servicios y las preferencias de los consumidores.

En lo que respecta a las empresas de servicios, en comparación con las empresas relacionadas con la fabricación, hay menos investigación sobre innovación y desempeño; sin embargo, existe evidencia empírica de que existe una correlación positiva entre innovación y desempeño en este sector económico. (Martinez Santa et al., 2010). En este sentido, cabe señalar que el concepto de la palabra innovación está más relacionado con la manufactura en sus orígenes, y se debe desarrollar y probar su importancia y aplicabilidad en el sector servicios. (Defelipe, Serpa, Castiblanco, \& Cardona, 2013), lo anterior permite plantear la siguiente hipótesis:

\section{$\mathrm{H}_{3}$ : El Factor Interno De La Innovación Tiene Una Influencia Positiva En La Ventaja Competiti- va De Las Organizaciones Del Sector Hotelero.}

El factor de Calidad, en la literatura también se considera como una explicación que incide directamente en la competitividad de la empresa en el mercado. (Camelo, Lorenzo, Martín, \& Valle, 1999); (Ramírez-Fernández et al., 2019). En este sentido, estudios que analizan la calidad de producto/servicio (García Garazao, Benito Torres, \& Varela Neira, 2011) (Calvo \& López, 2003), consideran que la reputación es un concepto generador de ventajas competitivas, porque depende de la percepción de calidad del cliente.

Autores como Aragón \& Rubio (2005), señalan en relación a la calidad, que las organizaciones competitivas se caracterizan por tratar de alinear los atributos y conjuntos de características del producto o servicio con las necesidades y expectativas de los clientes.

En referencia al factor en el sector hotelero, existe una correlación positiva entre el compromiso con la gestión de la calidad y la competitividad. (Claver Cortés, Pereira Moliner, Tarí Guilló, \& Molina Azorín, 2006); considerando que la 
industria se encuentra en un entorno globalizado, los requisitos y la experiencia de los turistas son cada vez mayores, por lo que las empresas de viajes deben brindar productos y servicios de alta calidad (Poon, 1993); (Weiermair, 1996), dando origen a la siguiente hipótesis:

\section{$\mathrm{H}_{4}$ : El Factor Interno De La Calidad Tiene Una} Influencia positiva En La Ventaja Competitiva De Las Organizaciones Del Sector Hotelero.

Finalmente, el Recurso o talento humano, Como base para desarrollar una ventaja competitiva en el nuevo enfoque actual de la economía del conocimiento (DeNisi, Hitt, \& Jackson, 2003). Es precisamente en los nuevos escenarios que se caracterizan por los rápidos cambios en el contexto propiciados por el siglo XXI donde cobra mayor relevancia la valoración del conocimiento como atributo inherente de los recursos humanos. (Passos-Simancas \& Arias-Aragones, 2016).

Aragón \& Rubio (2005) expresan que Los recursos humanos de la empresa son el factor más coincidente observado en la literatura, como explicación del éxito, que distingue los conocimientos, habilidades y actitudes de los trabajadores.

Otros corrientes teóricas y empíricas reconocen el talento humano como uno de los elementos con mayor valor dentro de una organización (Simerly, 1997) y el único recurso que la competencia está en capacidad de replicar (Evans \& Lindsay, 1996), tomando en consideración elementos como el empoderamiento de los empleados, la mejora de la relación laboral y la medición del desempeño como criterios importantes en el factor.

En este marco, el reto para las empresas es la gestión de recursos humanos, teniendo en cuenta la heterogeneidad de los mismos y los diferentes liderazgos estratégicos asociados, lo que deja patente la necesidad de una política adecuada de gestión y liderazgo de recursos humanos que promueva personal calificado y motivado, políticas de retribución y formación entre otros (Aragón \& Rubio, 2005).

Específicamente en el caso de las empresas hoteleras autores como (Loncica, Lavinia, Nicoleta, \& Loncica) citando a (Lashley, 2001; Mullins, 2001; Failte Ireland, 2005) plantean que Las personas son el centro de todos los aspectos de las actividades de la industria hotelera, desde la creación y el diseño hasta el desarrollo y la prestación de servicios, dando origen a la siguiente hipótesis:

\section{$\mathrm{H}_{5}$ : El Factor Interno De Recurso / Talento Hu-} mano Tiene Una Influencia positiva En La Ventaja Competitiva De Las Organizaciones Del Sector Hotelero.

En síntesis, resulta es evidente la distinción de los factores de marketing, capacidades directivas, innovación, calidad, y recursos humanos como fuentes impulsoras de ventajas competitivas en las organizaciones, generadora de rentas y mejoras en el rendimiento y desempeño. Estudios demuestran que solo cuando una empresa puede concretar sus competencias puede ser más competitiva en el mercado. Por lo tanto, integrar sus áreas funcionales permite examinar la relación entre fuentes o recursos de competencias y el desempeño organizacional (Li, 2000).

\section{Metodología}

Bajo un enfoque cuantitativo, se plantea una investigación de corte correlacional, con la finalidad de determinar el grado de relación causal entre variables, siendo estos los factores de competitividad empresarial de los hoteles del departamento de Sucre sobre la competitividad de los mismos, con el propósito de evaluar la relación que existe entre dos o más conceptos, categorías o variables en un contexto específico (Hernández Sampieri, Fernández Collado, \& Baptista Lucio, Pilar, 2004).

Los datos se obtuvieron a través de una encuesta aplicadas empresas hoteleras del departamento de Sucre, con registro Nacional de Turismo (RNT) activo a 1 marzo de 2019, debidamente registradas ante la Cámara de Comercio de Sincelejo. El cuestionario fue aplicado de manera presencial a gerentes y/o administradores de las empresas hoteleras del departamento obteniendo 60 respuestas.

Los factores internos asociados con la competitividad de las organizaciones fueron medidos a través de una escala propuesta por Martínez Santa et al., (2010) y Li, (2000) por medio de cuestionamientos con una escala de 1 a 5, comprendiendo valoraciones respecto a cada factor en donde 1 corresponde a el factor no es nada importante para la competitividad de la empresa, y 5 el factor es muy importante, como se observar en la siguiente tabla. 
Los Factores Claves de la Competitividad en el Sector Hotelero: una Propuesta desde la Teoría de los Recursos y Capacidades (VBR)

Tabla 2. Factores internos que generan competitividad

\begin{tabular}{|c|c|}
\hline Factor o fuente de competitividad & Ítem \\
\hline \multirow{4}{*}{ Factor de Marketing } & Reputación de la empresa \\
\hline & $\begin{array}{l}\text { Vigilancia del mercado: conocimiento del cliente y de la } \\
\text { competencia }\end{array}$ \\
\hline & Esfuerzo en marketing \\
\hline & $\begin{array}{l}\text { Capacidad de ofrecer paquetes y soluciones completas al } \\
\text { cliente }\end{array}$ \\
\hline \multirow{3}{*}{ Factor de Dirección } & Cualificación de los directivos \\
\hline & Visión Estratégica de los directivos \\
\hline & Innovación en dirección y gestión \\
\hline \multirow{4}{*}{ Factor de Innovación } & Innovación radical en servicio \\
\hline & Innovación incremental en servicio \\
\hline & Innovación en proceso productivo y comercial \\
\hline & $\begin{array}{l}\text { Colaboración con Universidades y Centros Tecnológicos } \\
\text { (como vía para la innovación) }\end{array}$ \\
\hline \multirow{2}{*}{ Factor de Calidad } & Calidad subjetiva del servicio (reputación del servicio) \\
\hline & Calidad del servicio \\
\hline \multirow{7}{*}{ Factor de Talento Humano } & Mejoras en la flexibilidad de las tareas de los empleados \\
\hline & Aumento en la formación laboral para trabajadores \\
\hline & $\begin{array}{l}\text { Aumento en la autonomía de los empleados en la realización } \\
\text { de sus trabajos }\end{array}$ \\
\hline & Relación empleado - gerencia \\
\hline & Desarrollo de criterios de medida de desempeño \\
\hline & $\begin{array}{l}\text { Sistemas de compensación y reconocimiento basado en } \\
\text { rendimiento }\end{array}$ \\
\hline & Establecimiento de manual de funciones \\
\hline
\end{tabular}

Fuente: elaboración propia con base a Martínez Santa et al., (2010) y Li (2000).

Las herramientas de validación estadísticas como el Alpha de Cronbach fueron planteadas en cada dimensión, y se espera que produzca un valor superior a 0,7 . Además se plantean otras herramientas estadísticas de análisis factorial exploratorio como la prueba K-M-O y la prueba de la esfera de Bartlett para validar el modelo propuesto. Posteriormente, se determinarán las cargas factoriales asociadas a cada escala, esto permitirá verificar si los ítems tienen relación con la escala a la que están asociadas teóricamente. Para la validación de estas cargas factoriales se tendrán en cuenta aquellas mayores a 0.5 .

\section{Resultados}

Como prueba preliminar para plantear el análisis factorial confirmatorio que permita validar las dimensiones de los factores de los recursos y capacidades internos respecto a la competitividad del sector hotelero en el Departamento de Sucre, se recurre al Alpha de Cronbach para garantizar la consistencia de los datos obtenidos, la siguiente tabla muestra el resultado global además del resultado individual de cada dimensión propuesta.

Tabla 3. Alpha de Cronbach

\begin{tabular}{cc}
\hline \hline Factores & Alpha de Cronbach \\
\hline Global &, 906 \\
Factor de Marketing &, 664 \\
Factor de Dirección &, 760 \\
Factor de Innovación &, 876 \\
Factor de Calidad &, 636 \\
Factor de Talento Humano &, 862 \\
\hline \hline
\end{tabular}


De acuerdo con los resultados, en términos generales el modelo muestra un buen ajuste en lo referido a la consistencia de los datos, puesto que el Alpha de Cronbach global demuestra una cifra muy cercana a uno lo cual es ideal para el análisis factorial confirmatorio e indica la viabilidad de esta herramienta estadística. En el apartado individual, son los factores de innovación y talento humano aquellos que demuestran mayor consistencia seguidos del factor de dirección, Marketing y en última instancia Calidad.

Otra herramienta estadística preliminar sugerida para validar el uso del análisis factorial con- firmatorio son las pruebas Kaiser-Meyer-Olkin (KMO) y esfericidad de Bartlett. La primera se asocia con las correlaciones entre las variables dando valores entre cero y uno en donde el uno implica una correlación fuerte entre estas siendo este resultado deseable para el análisis factorial confirmatorio. La segunda también se vincula con la correlación de las variables, sin embargo, la prueba de esfericidad de Bartlett es un estadístico al uso y posee una probabilidad que permite rechazar o validar la hipótesis de las correlaciones. Los resultados de estas pruebas se pueden observar en la siguiente tabla.

Tabla 4. Prueba KMO y esfericidad de Bartlett.

\begin{tabular}{ccccc}
\hline Factores & KMO & $\begin{array}{c}\text { Esfericidad } \\
\text { de Bartlett }\end{array}$ & gI & Sig. \\
\hline \hline Factor de Marketing &, 689 & 39,322 & 6 &, 000 \\
Factor de Dirección &, 677 & 50,199 & 3 &, 000 \\
Factor de Innovación &, 748 & 150,386 & 6 &, 000 \\
Factor de Calidad &, 500 & 14,873 & 1 &, 000 \\
\hline Factor de Talento Humano &, 813 & 215,378 & 21 &, 000 \\
\hline \hline
\end{tabular}

Los resultados respaldan el uso del análisis factorial confirmatorio en la medida en que la significancia de la prueba de esfericidad de Bartlett es inferiores a 0,05 mientras que los resultados de KMO demuestran correlaciones entre 0,5 y ,08. Esta es la importancia de complementar ambas pruebas debido a que en primera instancia los resultados del KMO distantes de 1 podrían incapacitar el uso del análisis factorial confirmatorio, sin embargo, la significancia de la prueba de Bartlett garantiza que estadísticamente los factores se encuentran correlacionados.

Los análisis preliminares anteriores apuntan al uso del análisis factorial confirmatorio para validar las dimensiones de los factores de los recursos y capacidades internos respecto a la competitividad del sector hotelero en el Departamento de Sucre, este análisis se puede observar en la siguiente tabla.

Tabla 5. Análisis Factorial Confirmatorio

\begin{tabular}{|c|c|c|c|}
\hline Dimensión & $\begin{array}{l}\text { Carga } \\
\text { Factorial }\end{array}$ & Ítem & $\begin{array}{l}\text { Carga Factorial } \\
\text { (Individual) }\end{array}$ \\
\hline \multirow{4}{*}{ Factor de Marketing } & $12,24 \%$ & Reputación de la empresa & 0,60 \\
\hline & & $\begin{array}{l}\text { Vigilancia del mercado: conocimiento } \\
\text { del cliente y de la competencia }\end{array}$ & 0,59 \\
\hline & & Esfuerzo en marketing & 0,65 \\
\hline & & $\begin{array}{l}\text { Capacidad de ofrecer paquetes y } \\
\text { soluciones completas al cliente }\end{array}$ & 0,47 \\
\hline \multirow{3}{*}{ Factor de Dirección } & $10,24 \%$ & Cualificación de los directivos & 0,79 \\
\hline & & Visión Estratégica de los directivos & 0,73 \\
\hline & & Innovación en dirección y gestión & 0,69 \\
\hline \multirow{4}{*}{ Factor de Innovación } & $26,20 \%$ & Innovación radical en servicio & 0,91 \\
\hline & & Innovación incremental en servicio & 0,82 \\
\hline & & $\begin{array}{l}\text { Innovación en proceso productivo y } \\
\text { comercial }\end{array}$ & 0,71 \\
\hline & & $\begin{array}{c}\text { Colaboración con Universidades y } \\
\text { Centros Tecnológicos (como vía para la } \\
\text { innovación) }\end{array}$ & 0,80 \\
\hline
\end{tabular}




\begin{tabular}{|c|c|c|c|}
\hline \multirow{2}{*}{ Factor de Calidad } & $8,25 \%$ & $\begin{array}{l}\text { Calidad subjetiva del servicio } \\
\text { (reputación del servicio) }\end{array}$ & 0,69 \\
\hline & & Calidad del servicio & 0,68 \\
\hline \multirow{8}{*}{$\begin{array}{l}\text { Factor de Talento } \\
\text { Humano }\end{array}$} & $28,12 \%$ & $\begin{array}{l}\text { Mejoras en la flexibilidad de las tareas } \\
\text { de los empleados }\end{array}$ & 0,34 \\
\hline & & $\begin{array}{l}\text { Aumento en la formación laboral para } \\
\text { trabajadores }\end{array}$ & 0.92 \\
\hline & & Aumento en la autonomía de los & 0,59 \\
\hline & & empleados en la realización de sus trabajos & \\
\hline & & Relación empleado - gerencia & 0,90 \\
\hline & & $\begin{array}{l}\text { Desarrollo de criterios de medida de } \\
\text { desempeño }\end{array}$ & 0,80 \\
\hline & & $\begin{array}{l}\text { Sistemas de compensación y } \\
\text { reconocimiento basado en rendimiento }\end{array}$ & 0,80 \\
\hline & & Establecimiento de manual de funciones & 0,80 \\
\hline Total & $85,05 \%$ & - & - \\
\hline
\end{tabular}

De acuerdo con los resultados obtenidos, los factores internos basados en la VBR desde un enfoque normativo explican en un $85,05 \%$ la competitividad del sector hotelero en Sucre, en donde los factores de talento humano e innovación ocupan un $54,32 \%$ indicando que estos elementos son fundamentales para la creación de competitividad en estas organizaciones. En el ámbito del talento humano las cargas factoriales más representativas corresponden a la relación empleado - gerencia, Desarrollo de criterios de medida de desempeño, Sistemas de compensación y reconocimiento basado en rendimiento y Establecimiento de manual de funciones. Por su parte, en el ámbito de la innovación los ítems con mayor carga factorial obedecen a Innovaciones radicales en servicio y la innovación incremental en servicio.

Por otra parte, las dimensiones de marketing, dirección y en menor medida la calidad demostraron ser factores generadores de competitividad secundarios ocupando el 30,73\% restante del modelo.

\section{Conclusiones}

La competitividad ocupa uno de los núcleos centrales de discusión, tanto en la academia como en el ámbito empresarial, respecto a cuáles son los factores que inciden en esta y permitiendo que las organizaciones puedan diferenciarse de la competencia, generando unas ventajas competitivas en el mercado. En ese contexto, la VBR surge como una corriente teórica que fundamenta las fuentes de competitividad en habilidades y dimensiones internas de la organización, entre es- tas, se han expuesto que son los factores de marketing, dirección, innovación, calidad y talento humano los que podrían incidir de forma directa en la competitividad de las organizaciones dedicadas al sector hotelero.

Bajo este marco, se planteó un estudio cuantitativo que, desde un enfoque formativo, permitiera establecer cuáles de estos factores son claves que inciden en la competitividad para el sector hotelero en Sucre. Posteriormente, se plantearon unas pruebas estadísticas preliminares que apoyaron el uso del análisis factorial confirmatorio arrojando como resultado que estos factores explican un $85 \%$ de la competitividad del sector hotelero en Sucre, además de confirmar que son las dimensiones de Talento Humano y la innovación los elementos claves para esta competitividad y en menor medida, factores como el marketing, la dirección y la calidad.

Estos resultados son coherentes tanto con la teoría como con trabajos empíricos anteriores (Martínez Santa et al., 2010; Li, 2000) estableciendo la importancia que tiene el talento humano y la innovación de forma específica en el sector hotelero proponiendo dos discusiones. La primera está referida al papel del talento humano dentro del servicio de hoteles, puesto que al analizar este factor interno por parte de la gerencia, en algunos casos no son tenidos en cuenta para el fortalecimiento de la organización, específicamente, se demostró por medio de herramientas estadísticas validadas que son elementos como; la relación empleado - gerencia; Desarrollo de criterios de medida de desempeño; Sistemas de compensación y 
reconocimiento basado en rendimiento; y Establecimiento de manual de funciones los que podrían conferir ventajas competitivas a los establecimientos de este sector. La segunda discusión se trata del papel de la innovación en las organizaciones hoteleras, de forma específica, aquellas vinculadas con nuevas formas de ofrecer los servicios, es decir, buscar la forma de brindar nuevas experiencias o generar valor introducción innovaciones dentro del servicio.

\section{Referencias}

Aldás, J., \& Uriel, E. (2017). Análisis multivariante aplicado con R. Madrid: Paraninfo S.A.

Álvarez, M. (2003). Competencias centrales y ventaja competitiva: el concepto, su evolución y su aplicabilidad. Contaduría y Administración, núm. 209, abril-junio UNAM, 5-22.

Aragón, A., \& Rubio, A. (2005). Factores explicativos del éxito competitivo: el caso de las PyMEs del estado de Veracruz. Contaduría y Administración. Universidad Nacional Autónoma de México,16, 35-69.

Aragón, A., \& Rubio, A. (2005). Factores explicativos del éxito competitivo: el caso de las pymes del estado de Veracruz. Contaduría y administración. Núm 216.

Aragón, A., Rubio, A., Serna, A. M., \& Chablé, J. (2010). Estrategia y competitividad empresarial: un estudio en las MyPiMEs en Tabasco. Investigación y Ciencia, vol. 18, núm. 47, pp. 4-12.

Arbelo Álvarez, A., \& Pérez Gómez, P. (2001). La reputación empresarial como recurso estratégico: un enfoque de recursos y capacidades. Ponencia presentada al XI Congreso Nacional de Acede. Zaragoza.

Àvalos, F. (2009). Factores determinantes de la competitividad empresarial. Repositorio Académico UPC. Cuadernos de Investigación. Vol.1, No 7.

Baena, E., Sánchez, J., \& Montoya, O. (2003). El entorno empresarial y la teoría de las cinco fuerzas competitivas. Scientia et Technica Año IX, No 23.

Barney, J. (1991). Firms Resources and Sustained Competitive Adventage. Journal of Management. Vol. 17, No 1, 99 - 120.

Barney, J. (2017). "Resources, capabilities, core competencies, invisible assets and knowledge assets: label proliferation and theory development in the field of strategic management". En The SIMS Blackwell Handbook of Organizational Capabilities (págs. 422 - 426). Constance E. Helfat.

Bonales, J., Zamora, A., \& Ortíz, C. (2015). Variables e Índices de Competitividad de las Empresas Exportadoras, utilizando el PLS. Revista CIMEXUS Vol. X, No.2, págs. 13-32.

Camelo, C., Lorenzo, J., Martín, F., \& Valle, R. (1999). Competitividad regional y recursos intangibles: Un análisis comparado Andalucia-España. Servicio de Publicaciones de la Universidad de Cádiz.

Camisón Zornoza, C., Garrigós Simon, F., \& Palacios Marqués, D. (2007). Estrategias competitivas y desempeño empresarial: Estudio comparativo de los modelos de Robinson \& Pearce y Miles \& Snow en el sector hotelero. Investigaciones Europeas de Dirección y Economía de la Empresa (IEDEE), ISSN 1135-2523, Academia Europea de Dirección y Economía de la Empresa (AEDEM), Vigo, Vol. 13, Iss. 3, 161-182.

Camisón, C. (1997). La competitividad de la pyme industrial española: estrategia y competencia distintivas. Civitas.

Cardona, A. (2011). Estrategia basada en los recursos y capacidades. Criterios de evaluación y el proceso de desarrollo. Revista electrónica FORUM. No 4. ISSN: 2027-2146. 
Los Factores Claves de la Competitividad en el Sector Hotelero: una Propuesta desde la Teoría de los Recursos y Capacidades (VBR)

Carmona-De Ríos, C. (2018). ¿Cuál es la importancia de implementar estrategias en las organizaciones? Revista Científica Anfibios, 1(1). https://doi.org/10.37979/afb.2018v1n1.20

Cunha Callado, A., Cunha Callado, A., \& de Almeida Holanda, F. (2011). Caracterización del uso de indicadores de desempeño no financiero en el sector hotelero. Estudios y Perspectivas en Turismo. Vol. 20, 876 - 890 .

Cupani, M. (2012). Análisis de Ecuaciones Estructurales: concepto, etapas y ejemplo de aplicación. Revista tesis $\mathrm{N}^{\mathrm{o}} 1,186-199$.

Del Río Cortina, J., Cardona Arbeláez, D., \& Pérez Olascoaga, S. J. (2018). Estrategias diferenciadoras y generadoras de valor en economías emergentes. Caso. Saber, Ciencia y Libertad, 13(1), 133-144. https://doi.org/10.18041/2382-3240/saber.2018v13n1.2083

Gómez, B. (2016). Estrategias para el fortalecimiento de la innovación y la competitividad del turismo a través del análisis de la dinámica innovadora del Sistema Integrado de Innovación Sectorial en el departamento de Sucre. Cartagena.

Grant, R. M. (1991). The Resource Based Theory of Competitive Advantage: Implications for Strategy Formulation. California Management Review, 114 - 135.

Gupta, A., \& Govindarajan, V. (1984). Business unit strategy, managerials characteristics, and business unit efectitiveness at strategy implementation. Academy of Management Journal, Vol. $27,25-41$.

Hernández Sampieri, R., Fernández Collado, C., \& Baptista Lucio, Pilar. (2004). Metodología de la Investigación. Tercera edición. México: McGraw - Hill Interamericana.

Hill, C., Jones, G., \& Schilling, M. (2014). Strategic management: theory: an integrated approach. Cengage Learning.

Hu, L., \& Bentler, P. (1999). Criterios de corte para índices de ajuste en análisis de estructura de covarianza: convencional Criterios versus nuevas alternativas. SEM, 6(1), 1-55.

Huertas Riveros, P., Navas López, J., \& Almodóvar Martínez, P. (2004). La diversificación desde la Teoría de Recursos y Capacidades. Cuadernos de Estudios Empresariales, núm. 14, 87 - 104.

Ingram, H., \& Daskalakis, G. (1999). Measuring quality gaps in hotels: The case of Crete. International journal of comtemporar hospitality management, vol. 11, $\mathrm{n}^{\mathrm{o}} 1,24-30$.

Krugman, P. (1994). Competitiveness: A dangerous obsession. Foreign Affairs, vol. 73 NQ 2: 28-44.

Li, L. X. (2000). An analysis of sources of competitiveness and performance of Chinese manufacturers. : International Journal of Operations and Production Management, 20(3), 299-315. https://doi.org/10.1108/01443570010294307

Lippman, S. A., \& Rumelt, R. P. (1982). Uncertain imitability: an analysis of interfirm differences in efficiency under competition. The Bell Journal of Economics, vol. 13, núm. 2, 418-438.

Long, J. S. (1983). Confirmatory Factor Analysis: A Preface to LISREL. University Paper Series on Quantitative Applications in the Social Sciences. Newbury Park, CA: Sage: SAGE Publications.

Malhotra, N. K., \& Dash, S. (2011). Investigación de mercado y orientación aplicada. Londres: Pearson Publishing.

Martínez Santa, R., Charteriana Abando, J., \& Araujo de la Mata, A. (2010). Un modelo causal de competitividad empresarial planteado desde la VBR: Capacidades Directivas, Marketing, Innovación y Calidad. Investigaciones Europeas de Dirección y Economía de la Empresa. Vol. $16, N^{\circ} 2,165-188$, ISSN: 1135-2523. 
Mazaira Castro, A., Dopico Parada, A., \& González Vázquez, E. (2005). Incidencia del grado de orientación al mercado de las organizaciones empresariales en el desarrollo de las capacidades estratégicas de marketing. Revista Europea de Dirección y Economía de la Empresa vol. 14, núm. 3, 181 - 208.

Medina, R. (2013). La Ventaja Competitiva como elemento fundamental de la estrategia y su relación con el sector servicios de la actividad turística. Observatorio de la Economía Latinoamericana $\mathrm{N}^{\mathrm{o}} 191$.

Méndez Álvarez, C. E. (2001). Metodología: Diseño y Desarrollo del Proceso de Investigación.

Navarro, M., \& Minondo, A. (1999). Competitividad y empleo en la industria manufacturera: un análisis comparado. Ekonomiaz: Revista Vasca de Economia, núm. 44, pp. 92-115.

Nelson, R. (1992). Recent Writings on Competitiveness: Boxing the Compass. California Management Review, Vol. 34, No. 2, 127-137.

Newbert, S. L. (2008). Value, rareness, competitive advantage,and performance: a conceptual level empirical investigation of the resource based view of the firm. Strategic Management Journal, 745-768.

OCDE. (2005). Manual de Oslo. Guía para la recogida e interpretación de datos sobre innovación, $3^{\mathrm{a}}$ ed. París: OCDE y EUROSTAT.

Oficina de Estudios Económicos. (2018). Perfiles Económicos Departamentales - Departamento de Sucre. Bogotá.

Passos-Simancas, E. S., \& Arias-Aragones, F. J. (2016). El capital humano como factor de competitividad en la industria hotelera y turística en Cartagena. Panorama Económico, Vol 24, 269-282.

Pelham, A. M. (1997). Mediating influences on the relations between market orientation and profitability in small industrial firms. Journal of Marketing Theory and Practice, 5(3), 55-76.

Penrose, E. (1959). The theory of the Growth of the Firm. New York, United States: Oxford university press.

Ramírez-Fernández, R., Machado-Licona, J., \& Fernández-Ramírez, O. (2019). Calidad en la educación universitaria, desde el programa de ingeniería de sistemas: una visión cualitativa de la educación superior. Revista Científica Anfibios, 2(2), 41-50. https://doi.org/10.37979/ afb. $2019 \mathrm{v} 2 \mathrm{n} 2.49$

Ruizalba Robledo, J. L., Vallespín Arán, M., \& Pérez-Aranda, J. (2015). Gestión del conocimiento y orientación al marketing interno en el desarrollo de ventajas competitivas en el sector hotelero. Investigaciones Europeas de Dirección y Economía de La Empresa, 21(2), 84-92. https://doi.org/10.1016/j.iedee.2014.03.001 